\title{
Pathology Reporting of Thyroid Core Needle Biopsy: A Proposal of the Korean Endocrine Pathology Thyroid Core Needle Biopsy Study Group
}

Chan Kwon Jung ${ }^{1} \cdot$ Hye Sook Min ${ }^{2,3}$ Hyo Jin Park ${ }^{2}$. Dong Eun Song ${ }^{4}$ Jang Hee Kim ${ }^{5}$. So Yeon Park ${ }^{2}$ Hyunju Yoo ${ }^{6}$. Mi Kyung Shin ${ }^{7}$ Korean Endocrine Pathology Thyroid Core Needle Biopsy Study Group

${ }^{1}$ Department of Hospital Pathology, College of Medicine, The Catholic University of Korea, Seoul; ${ }^{2}$ Department of Pathology, Seoul National University College of Medicine, Seoul; ${ }^{3}$ Department of Epidemiology and Preventive Medicine, Graduate School of Public Health, Seoul National University, Seoul; ${ }^{4}$ Department of Pathology, University of Ulsan College of Medicine, Seoul; ${ }^{5}$ Department of Pathology, Ajou University School of Medicine, Suwon; ${ }^{6}$ Department of Pathology, Daerim Saint Mary's Hospital, Seoul; ' Department of Pathology, Hallym University College of Medicine, Seoul, Korea

Received: April 16, 2015

Revised: June 2, 2015

Accepted: June 3, 2015

Corresponding Authors

Chan Kwon Jung, MD, PhD

Department of Hospital Pathology, Seoul St. Mary's Hospital, College of Medicine, The Catholic

University of Korea, 222 Banpo-daero, Seocho-gu,

Seoul 137-701, Korea

Tel: +82-2-2258-1622

Fax: +82-2-2258-1627

E-mail: ckjung@catholic.ac.kr

Mi Kyung Shin, MD, PhD

Department of Pathology, Hallym University

Kangnam Sacred Heart Hospital, Hallym University College of Medicine, 1 Singil-ro, Yeongdeungpo-gu, Seoul 150-950, Korea

Tel: +82-2-829-5443

Fax: +82-2-829-5268

E-mail:smk0103@yahoo.co.kr
In recent years throughout Korea, the use of ultrasound-guided core needle biopsy (CNB) has become common for the preoperative diagnosis of thyroid nodules. However, there is no consensus on the pathology reporting system for thyroid CNB. The Korean Endocrine Pathology Thyroid Core Needle Biopsy Study Group held a conference on thyroid CNB pathology and developed guidelines through contributions from the participants. This article discusses the outcome of the discussions that led to a consensus on the pathology reporting of thyroid CNB.

Key Words: Thyroid nodule; Guideline; Image-guided biopsy; Preoperative period; Diagnosis

Ultrasound-guided fine needle aspiration cytology (FNAC) is the most accurate, effective and safe method for the preoperative assessment of thyroid nodules. ${ }^{1}$ Since 2007 , pathologists and clinicians have widely adopted the six-level diagnostic scheme of
The Bethesda System for Reporting Thyroid Cytopathology (TBSRTC). The use of standard diagnostic categories has a significant clinical benefit in terms of risk stratification for malignancy, and enhances communication between pathologists and physi- 
cians. ${ }^{2,3}$ However, a major limitation of FNAC for the diagnosis of thyroid nodules, is the relatively high incidence of nondiagnostic aspirates $(10 \%-15 \%)$ and indeterminate aspirates $(10 \%-$ $30 \%){ }^{4,5}$ Patients with thyroid nodules of nondiagnostic or indeterminate aspirates typically undergo repeated aspirations for FNAC or diagnostic surgery. ${ }^{1}$

Ultrasound-guided core needle biopsy (CNB) has been successfully employed for the last decade as a complementary tool for the evaluation of thyroid nodules. ${ }^{6-9} \mathrm{CNB}$ reportedly reduces the rate of nondiagnostic or indeterminate results. ${ }^{6}$ Therefore,
CNB can be used when FNAC results are nondiagnostic or fail to correlate with clinical findings or results of ultrasound imaging studies. ${ }^{4,8,10,11}$ Limited data suggest that $\mathrm{CNB}$ can be used as a first-line method for the preoperative diagnosis of thyroid nodules with a high risk of malignancy. ${ }^{12}$ Thyroid CNB also allows ancillary immunohistochemical and molecular tests to be carried out on the tissue sample.

Ultrasound-guided thyroid CNB is a safe procedure in contrast to large $\mathrm{CNB}$, which was employed in the 1990 s and requires the use of a larger diameter needle. ${ }^{6}$ The overall complication
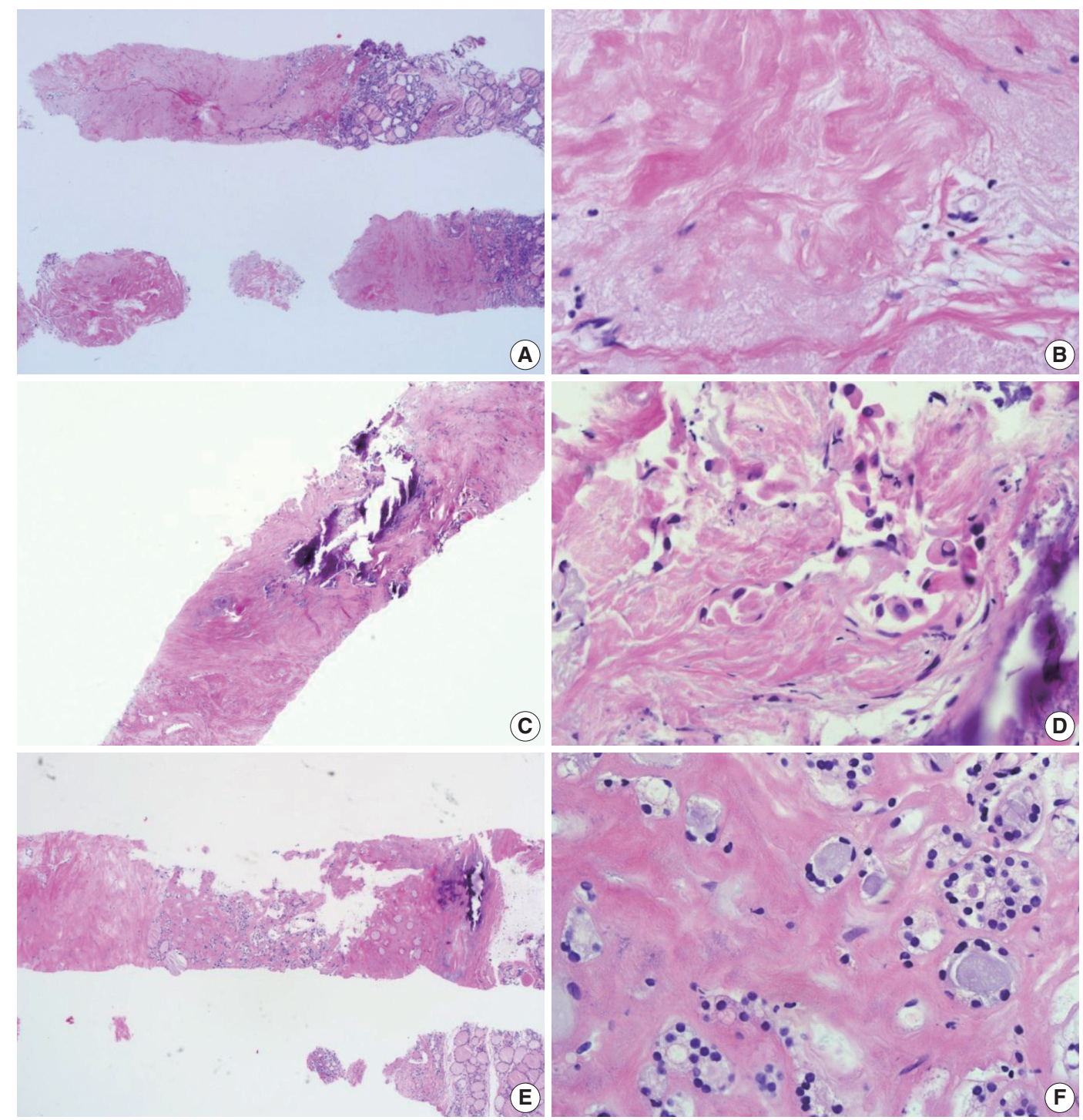

Fig. 1. Core needle biopsies of fibrotic nodules. The right column images represent the high-power views of the lesional area in the left column images. (A) The specimen consists of an acellular fibrotic lesion and adjacent normal parenchyma. (B) The fibrotic area contains no follicular cells, but contains a few lymphocytes and stromal cells. This lesion is classified in the nondiagnostic category. (C) The specimen shows a paucicellular structure with marked fibrosis and calcification. (D) Scattered atypical cells with suspicious morphological features of papillary carcinoma are embedded in the fibrosis. This lesion contains suspicious follicular cells and should therefore be diagnosed as suspicious for malignancy or as a malignancy, depending on the degree of nuclear atypia. (E) The specimen shows marked fibrosis and calcification. (F) The highpower view of the lesion shows relatively numerous benign-appearing follicular cells. This lesion can be diagnosed as a benign follicular nodule. 
rate is similar to what has been reported for FNAC., ${ }^{6,9,13}$

The main goal of histopathologic examination by $\mathrm{CNB}$ is to triage patients with thyroid disease who need to be surgically managed. Through the use of $\mathrm{CNB}$, a definitive diagnosis is possible in most, but not necessarily all patients. These concepts are the same as those of FNAC. However, in contrast to FNAC, there is no consensus on the diagnostic criteria for reporting thyroid $\mathrm{CNB}$.

\section{CONSENSUS CONFERENCE}

Since May 2013, the Korean Endocrine Pathology Thyroid Core Needle Biopsy Study Group has held three consensus conferences on thyroid CNB pathology reporting in Seoul, Korea. The purpose of the conferences was to bring together endocrine pathologists who were thyroid specialists (1) to review existing reporting formats and published literature related to thyroid
CNB, (2) to review 25 thyroid CNB specimens using virtual microscopy on each slide, (3) to reach a consensus regarding the histological diagnostic criteria and reporting format of thyroid CNB pathology, and (4) to develop a standard pathology reporting system for thyroid CNB that would be useful for clinical management and pathologic diagnosis. Twenty-one Korean endocrine pathologists participated in the conferences. In Korea, pathologists and clinicians are familiar with TBSRTC terminology and the management guidelines of each diagnostic category. ${ }^{3}$ Therefore, all participants in the conferences agreed to use the standardized diagnostic categories for thyroid CNB.

\section{CONTROVERSIAL SUBJECTS}

Most participants decided to use the same six-level system as TBSRTC for categorizing the results of thyroid CNB. However, the following concerns exist with regard to the use of the same

Table 1. Diagnostic categories of thyroid core needle biopsy

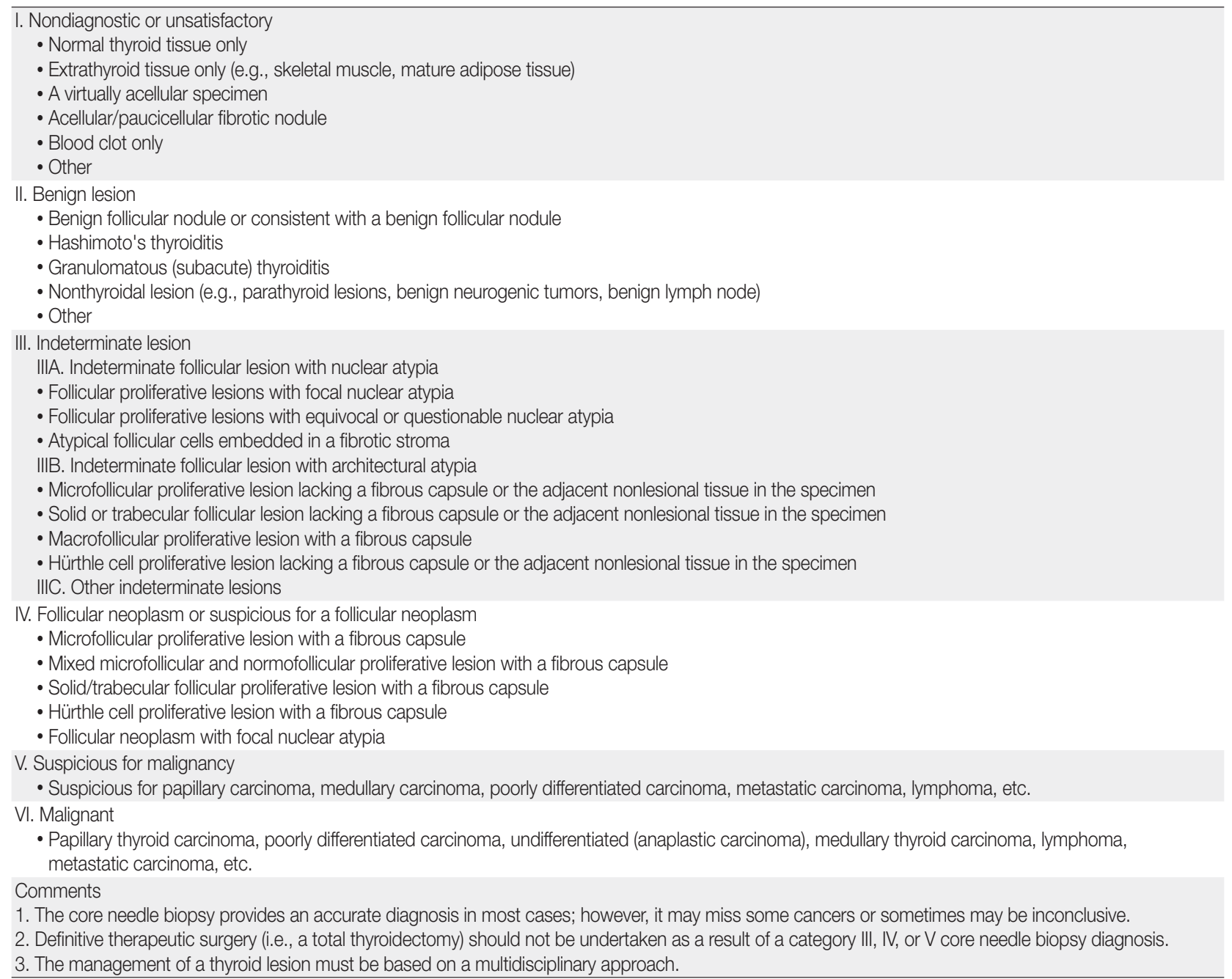


terminology: (1) some clinicians and pathologists may understand the meaning of terms in a CNB pathology report differently from the terms in an FNAC report; (2) the phrase "atypia of undetermined significance" in TBSRTC is inappropriate terminology in the field of histopathology; (3) there is no consensus regarding specimen adequacy; and (4) the sample representativity of the CNB may be different from that of the FNAC. Therefore, we recommend that the limitations of $\mathrm{CNB}$ should be explained in the pathology report.

Most participants in the conferences agreed that a sample composed primarily of an acellular/paucicellular fibrotic nodule should be considered nondiagnostic if it did not contain any atypical cells (Fig. 1). However, a categorical diagnosis should be rendered, irrespective of the number of follicular cells, if a sample contains atypical cells (Fig. 1).

Another dilemma is the differential diagnosis of follicular proliferative lesions without nuclear atypia in a CNB sample. Histologically, hyperplastic nodules are usually found in multiples and are partially encapsulated by a fibrous capsule. Follicular adenoma is a completely encapsulated follicular nodule that is mostly solitary and has microscopic features that are different from those of the surrounding normal thyroid tissue. ${ }^{14}$ Fibrous
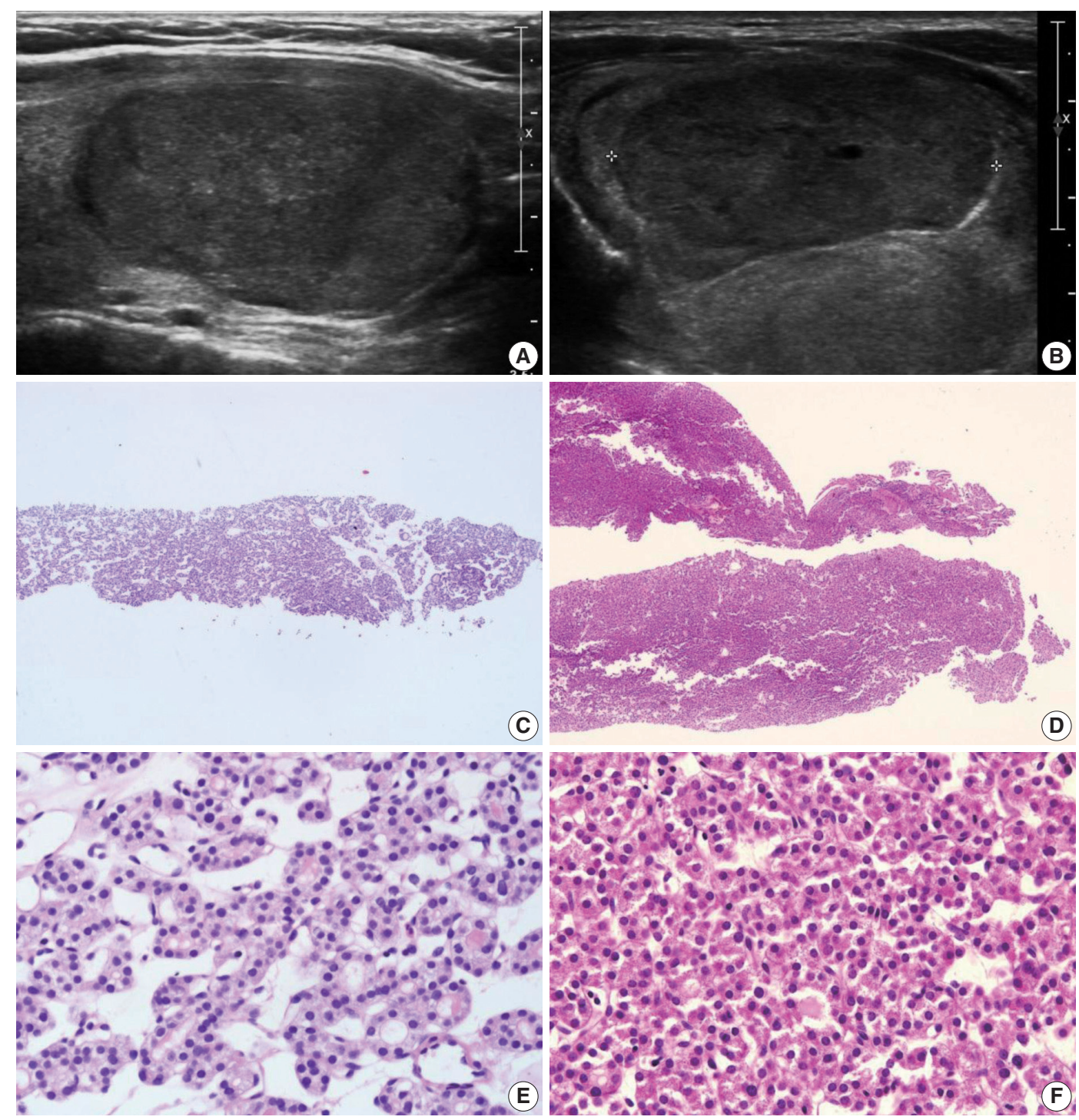

Fig. 2. (A, B) The ultrasound images show well-circumscribed solid, homogeneous, nodules with peripheral hypoechoic rims. (C, D) The core needle biopsies show only microfollicular proliferation. These specimens do not contain a fibrous capsule or adjacent normal tissue that is required to make a diagnosis of follicular neoplasm. (E, F) Images are the high-power views of Fig. 2C and D, respectively. No nuclear atypia is present. The left and right columns show the conventional and Hürthle cell types, respectively. Typical ultrasound features of follicular neoplasms, when present, can lead to the diagnosis of follicular neoplasms, even when specimens are not contained in a fibrous capsule. 
capsules of follicular neoplasms vary in thickness. Tumor capsules are usually thin on follicular adenomas and tend to be thicker on follicular carcinomas. ${ }^{14}$ It is not possible to differentiate between follicular adenoma and carcinoma using FNAC or CNB samples because the diagnosis of follicular carcinoma is based on the presence of tumor capsular invasion or vascular invasion. Nonetheless, in CNB samples, it may be possible to determine whether a nodule is a follicular neoplasm or a non-neo- plastic lesion. ${ }^{15}$ Most participants agreed that a diagnosis of "follicular neoplasm or suspicious for a follicular neoplasm" is appropriate when a follicular proliferative lesion is separated from the surrounding thyroid parenchyma by a definite fibrous capsule, has features that are architecturally and cytologically different from the adjacent thyroid glands, and lacks the nuclear features of a papillary carcinoma. However, the CNB specimen of a widely invasive follicular carcinoma may not show a fibrous
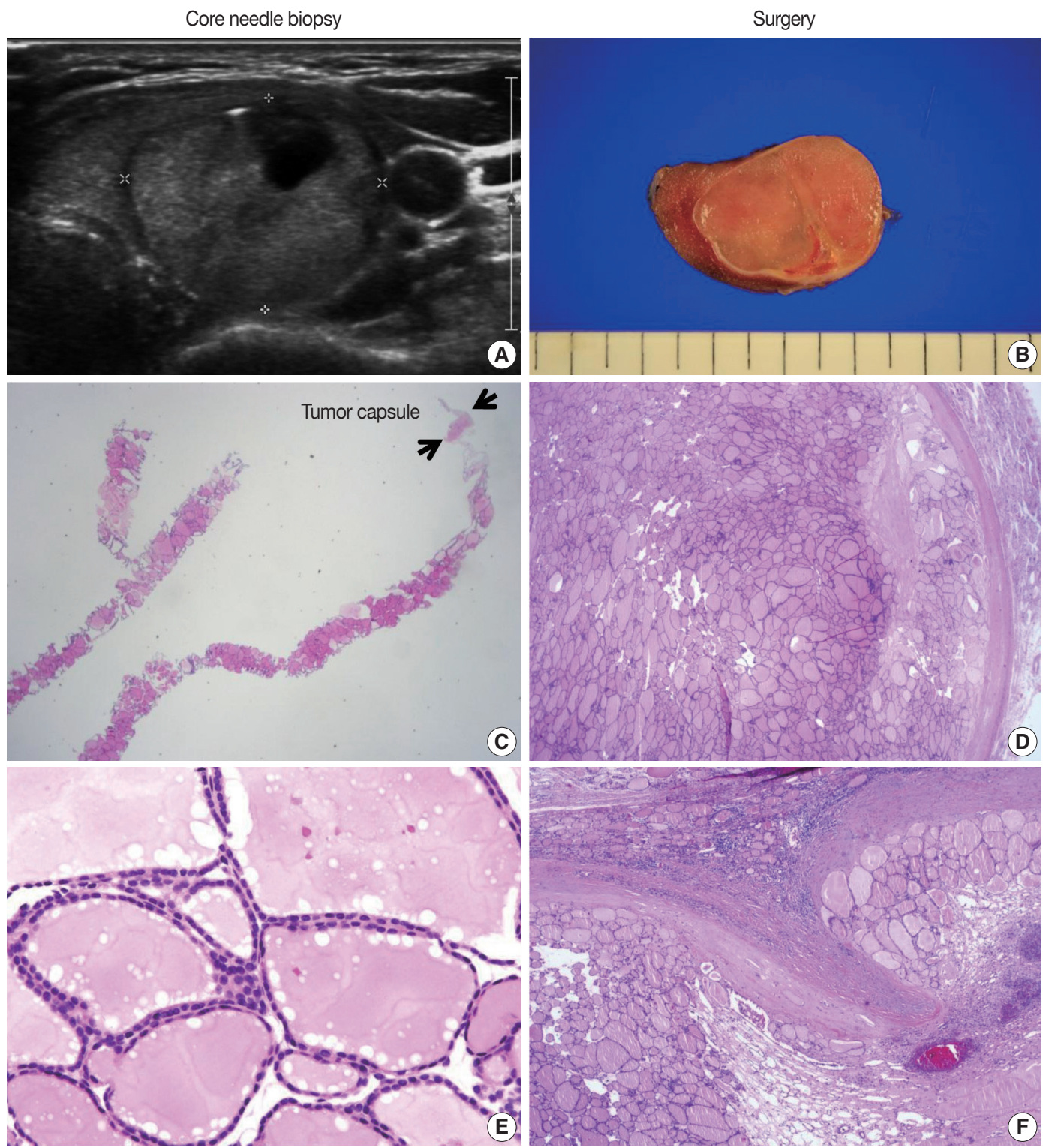

Fig. 3. Core needle biopsy findings of a follicular neoplasm with a macrofollicular growth pattern. The images in the left column and the right column show the core needle biopsy specimen and the resected specimen, respectively. (A) The ultrasound image shows a well-circumscribed, isoechoic, ovoid nodule with a peripheral hypoechoic rim. A focal cystic change is present. (B) The surgical specimen exhibits a thick fibrotic capsule surrounding the nodule. (C) The core needle biopsy shows a macrofollicular proliferative lesion with a fibrous capsule (arrows). (E) The high-power view of the biopsy specimen shows benign-appearing follicular cells. The typical ultrasound features and thick fibrous capsule can lead to a diagnosis of follicular neoplasm, even in a macrofollicular lesion. The microscopic examination of the surgical specimen shows that the tumor is well encapsulated (D) and capsular invasion is minimal (F). 
capsule because the tumor is not encapsulated although it has invaded into the thyroid parenchyma and extrathyroidal tissue. Some hyperplastic nodules have a cellular microfollicular or solid growth pattern and a fibrous capsule. Therefore, misdiagnosing a widely invasive follicular carcinoma as a benign follicular lesion or a hyperplastic nodule as a follicular neoplasm on the $\mathrm{CNB}$ specimens remains a concern.

No study has yet been conducted to define the number of tissue cores that are required for an accurate diagnosis of a thyroid nodule. However, most conference participants agreed that two cores are adequate to diagnose a lesion.

\section{CONSENSUS DIAGNOSTIC CATEGORIES}

In the histologic examination of CNB samples, a diagnostic category is selected from the following six standardized options. This proposal was approved by the Korean Endocrine Pathology Thyroid Core Needle Biopsy Study Group (Table 1).

\section{Nondiagnostic or unsatisfactory}

This category includes specimens with an insufficient number of follicular cells to provide an appropriate diagnosis or when the specimen is not representative of the ultrasound image of the lesion. This diagnosis is subjective; and therefore, the report should explain why the sample is nondiagnostic or unsatisfactory. For example, the sample may be normal thyroid tissue only, extrathyroid tissue only (e.g., skeletal muscle and mature adipose tissue), a virtually acellular specimen, an acellular/paucicellular fibrotic nodule, a blood clot only, or it may show other nondiagnostic or unsatisfactory findings. However, any CNB specimen containing atypical cells should not be considered nondiagnostic or unsatisfactory.

Normal thyroid tissue or extrathyroid tissue only implies that the thyroid lesion has not been sampled. For some benign follicular lesions with normofollicular structures, it may be difficult to assess specimen adequacy. It is important to find the transition between the follicular lesion and the surrounding normal parenchyma and compare between the pathologic finding and its ultrasound images.

\section{Benign}

This category includes all benign thyroidal and nonthyroidal diseases. A CNB specimen can be classified, based on the specific diagnosis for the lesion, as benign. For example, the sample may
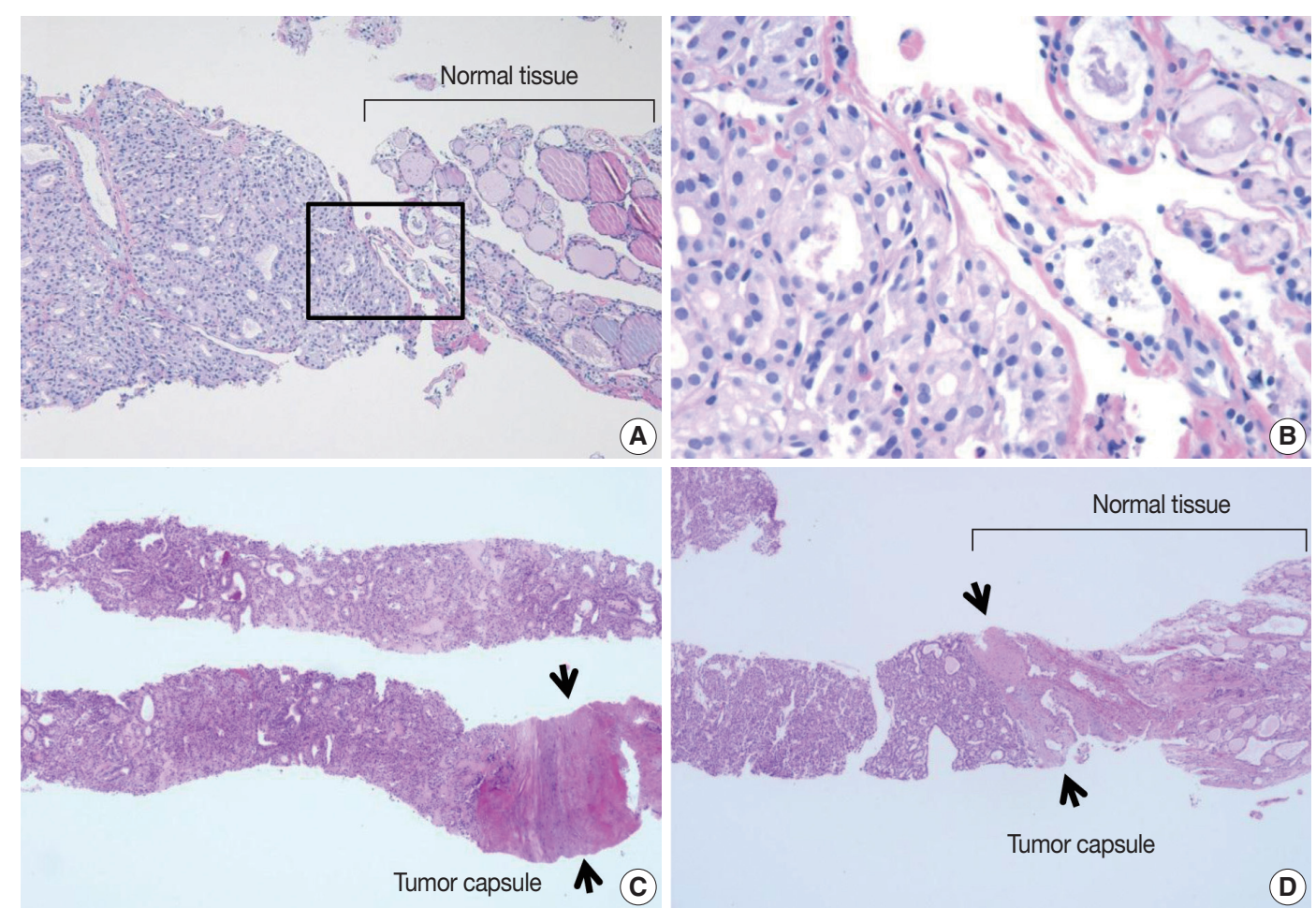

Fig. 4. (A) The core needle biopsy shows a microfollicular proliferative lesion and surrounding normal tissue. (B) The high-power view of the boxed area in Fig. 4A shows that the lesion has no nuclear atypia or fibrous capsule. This lesion should be diagnosed as a benign follicular nodule. (C, D) When microfollicular proliferative lesions show a definite fibrous capsule (arrows) in the core needle biopsy, the specimens should be diagnosed as a follicular neoplasm. 
be a benign follicular nodule or consistent with a benign follicular nodule, Hashimoto's thyroiditis, subacute granulomatous thyroiditis, a nonthyroidal lesion (e.g., a parathyroid lesion, benign neurogenic tumors, and benign lymph node), or another benign lesion.

A benign follicular nodule encompasses nodular hyperplasias (adenomatoid nodule), colloid nodules, a nodule in Graves' disease, nodular Hashimoto's thyroiditis, and a subset of follicular adenomas. CNB specimens of these lesions show a benign-ap- pearing normofollicular or macrofollicular structure and do not have a well-defined fibrous capsule.

\section{Indeterminate lesion}

The cytologic atypia and histologic growth patterns in this category are of uncertain significance and insufficient to be classified under other diagnostic categories. The diagnostic category III "indeterminate lesion" corresponds to "atypia of undetermined significance" or "follicular lesion of undetermined signif-
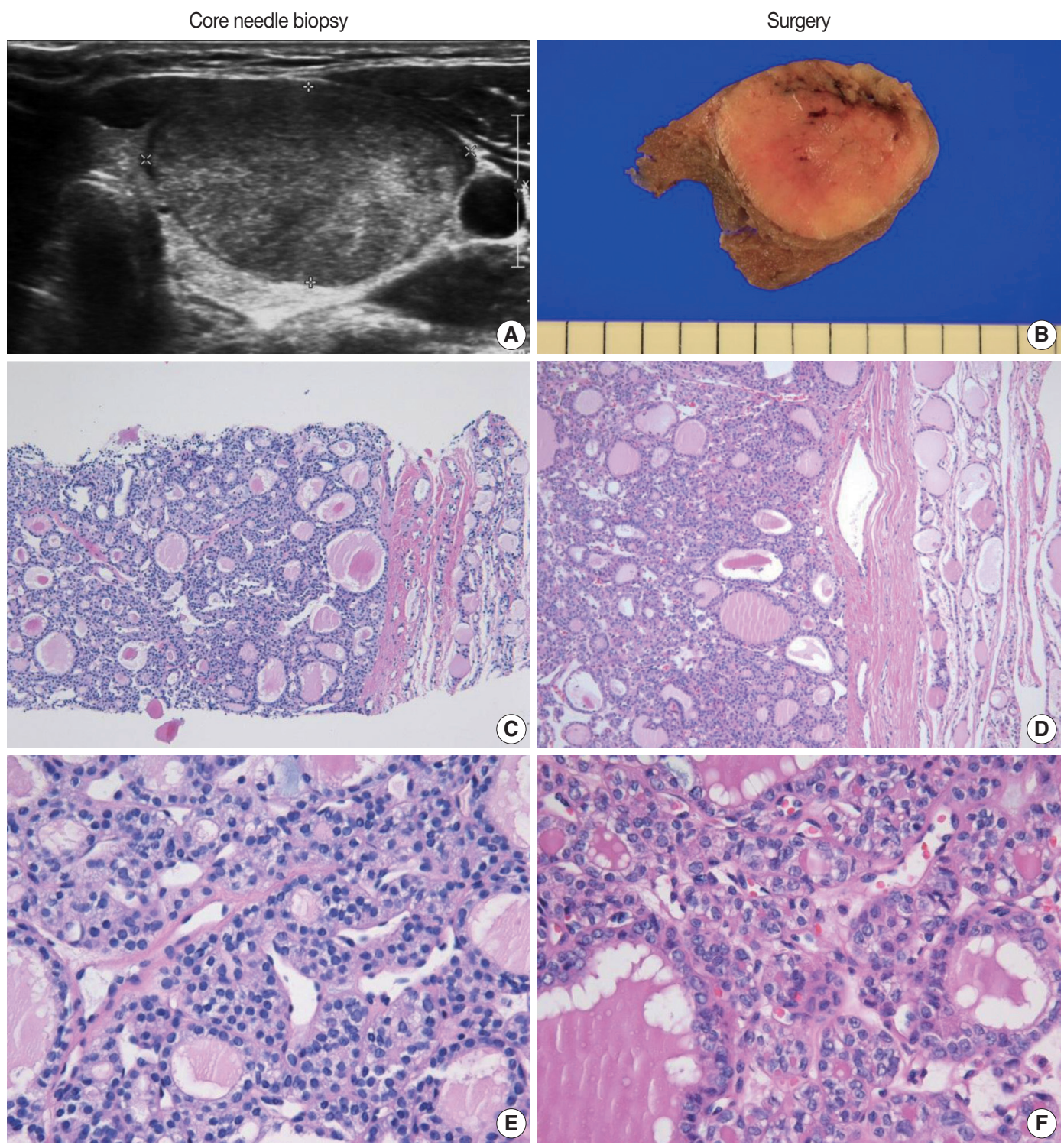

Fig. 5. The core needle biopsy of a follicular neoplasm with focal nuclear atypia. The images in the left and right columns show the findings of the core needle biopsy and the corresponding surgical specimen, respectively. (A) The ultrasound image shows a solid, homogeneous, hypoechoic, ovoid nodule with a peripheral halo. (B) The cut surface of the resected specimen corresponds to the ultrasound image in Fig. 5A. $(C, D)$ The low-power view shows a follicular proliferative lesion with a fibrous capsule. (E) The high-power view of Fig. $5 \mathrm{C}$ reveals focal nuclear atypia. $(F)$ The corresponding image in the surgical specimen more definitely shows the morphological features (e.g., nuclear enlargement, irregularity, clearing, and grooves) of a follicular variant of papillary carcinoma. 
icance" in TBSRTC. However, it was considered inappropriate to use the term "atypia of undetermined significance" in the histopathologic diagnosis of $\mathrm{CNB}$ specimens.

The diagnosis of category III is appropriate when a follicular proliferative lesion shows focal nuclear atypia such as nuclear enlargement with pale chromatin, irregular nuclear membranes, and nuclear grooves in a background of predominantly benignappearing follicles. If a microfollicular proliferative lesion is separated by a fibrous capsule from the surrounding normal parenchyma it is diagnosed as a follicular neoplasm (see category IV). If a fibrous capsule or adjacent nonlesional tissue is not identified in a CNB specimen that shows a predominantly microfollicular or trabecular growth pattern, it is reasonable to classify the lesion as diagnostic category III because it cannot be determined whether the nodule has a fibrous capsule. However, if sonographic features suggest a follicular neoplasm in such lesions, the sample can be considered a category IV, "follicular neoplasm" (Fig. 2). ${ }^{15}$ A multidisciplinary approach to a thyroid nodule can improve the preoperative diagnostic accuracy of FNAC and CNB specimens. ${ }^{16}$

FNAC specimens of macrofollicular lesions are usually diag- nosed as benign. However, when ultrasound images show the typical features of a follicular neoplasm and the $\mathrm{CNB}$ specimen microscopically shows a fibrous capsule, then the $\mathrm{CNB}$ specimen can be diagnosed as a follicular neoplasm, even in a macrofollicular lesion (Fig. 3). However, in surgical pathologies, most participants agreed that category III is appropriate in a macrofollicular proliferative lesion with a definite fibrous capsule (Fig. 3).

The following are common scenarios that may be encountered in case of follicular proliferative lesions:

IIIA. Indeterminate follicular lesion with nuclear atypia

Examples in this category are follicular proliferative lesions with focal nuclear atypia, follicular proliferative lesions with equivocal or questionable nuclear atypia, and atypical follicular cells embedded in fibrotic stroma.

IIIB. Indeterminate follicular lesion with architectural atypia

Examples in this category are microfollicular proliferative lesions lacking a fibrous capsule or the adjacent nonlesional tissue in the specimen; solid or trabecular follicular lesions lacking a fibrous capsule or the adjacent nonlesional tissue in the specimen; macrofollicular proliferative lesions with a fibrous capsule; Hürthle cell proliferative lesions lacking a fibrous capsule or the

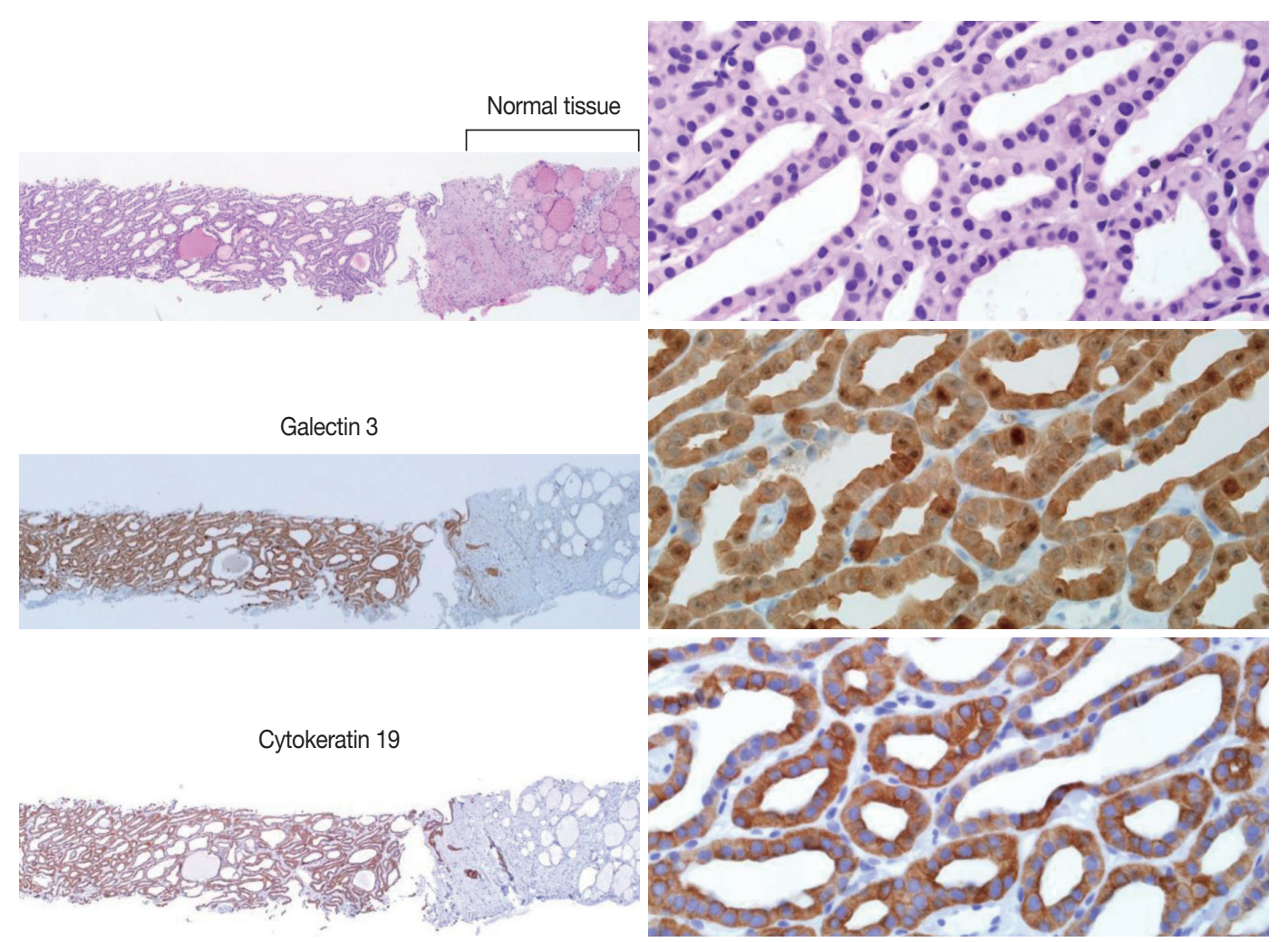

Fig. 6. The core needle biopsy shows a follicular proliferative lesion with nuclear atypia and diffuse strong immunohistochemical staining for galectin 3 and cytokeratin 19 in the tumor cells. Images in the left and right columns show the low magnification and high magnification views, respectively, of the samples. 
adjacent nonlesional tissue in the specimen.

IIIC. Other indeterminate lesions

\section{Follicular neoplasm or suspicious for a follicular neoplasm}

In CNB and FNAC, the term "follicular neoplasm or suspicious for a follicular neoplasm" is used to encompass neoplastic lesions with follicular proliferative patterns (e.g., follicular adenoma, follicular carcinoma, follicular variant of papillary carcino$\mathrm{ma}$, follicular variant of medullary carcinoma). ${ }^{2,3}$ The histologic diagnosis of "follicular neoplasm or suspicious for a follicular neoplasm" in a CNB specimen is based on the presence of a fibrous capsule and microscopic features that differ from the adjacent thyroid parenchyma (Fig. 4). Follicular cells do not show the typical nuclear features of papillary carcinomas. It is important to identify a well-formed fibrous capsule in the CNB specimen. The FNAC diagnosis for follicular neoplasm is primarily based on the presence of microfollicular or trabecular architecture and the lack of colloid. In a CNB specimen, the growth patterns of a follicular neoplasm can be microfollicular, normofollicular, solid, or trabecular when a fibrous tumor capsule is identified in the sample. Examples in this category include microfollicular proliferative lesions with fibrous capsules, mixed microfollicular and

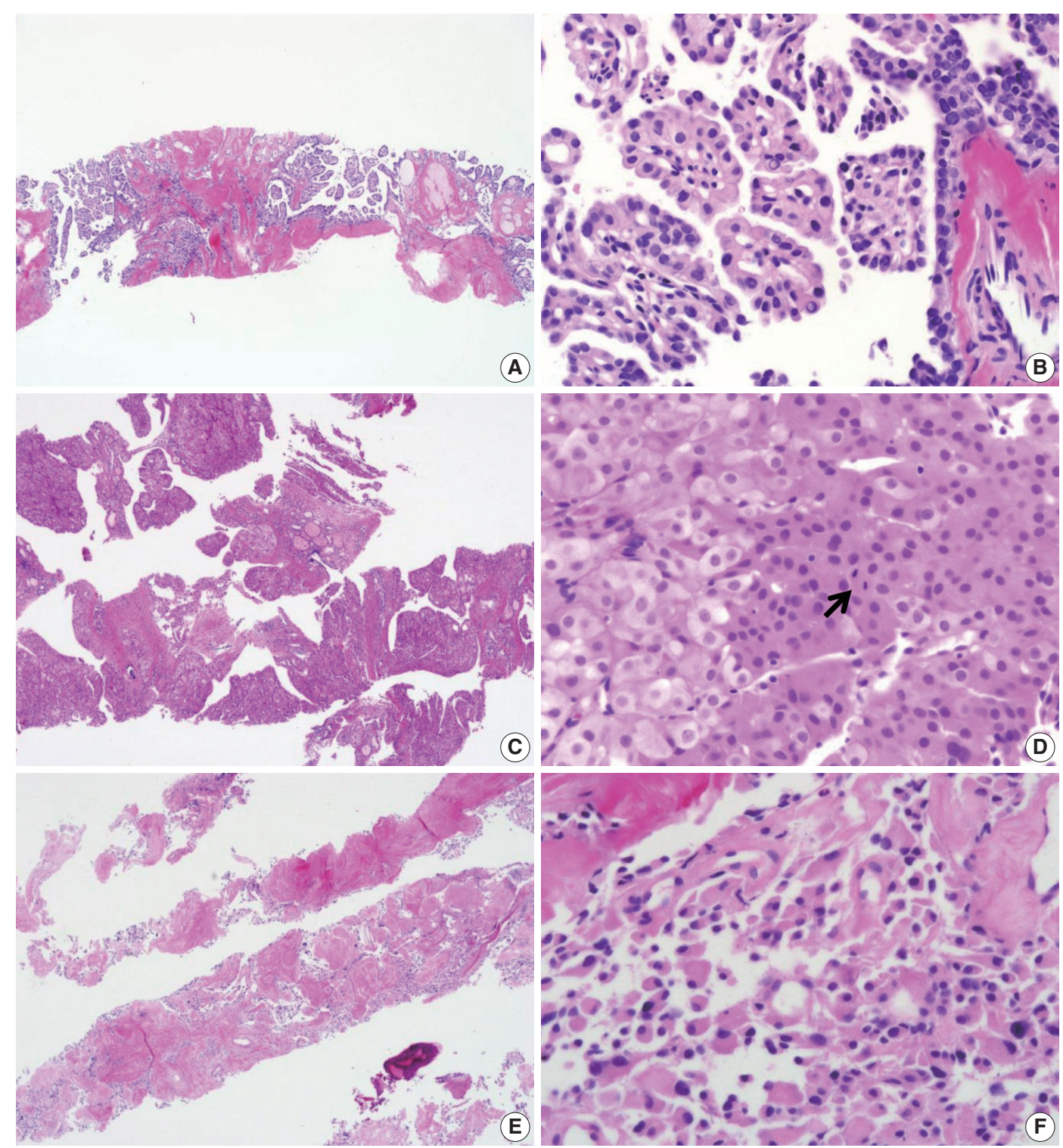

Fig. 7. Core needle biopsies of malignant thyroid nodules. (A, B) The biopsy specimen maintains the typical morphological features of papillary carcinoma. Poorly differentiated carcinoma shows solid, trabecular, and insular growth patterns (C) and mitosis (arrow) (D) under the highpower view. The medullary carcinoma shows the typical morphological features under the low-power view $(\mathrm{E})$ and the high-power view (F). 
normofollicular proliferative lesions with fibrous capsules, solid or trabecular follicular lesions with fibrous capsules, Hürthle cell proliferative lesions with fibrous capsules, and follicular neoplasms or samples suspicious for follicular neoplasms with focal nuclear atypia.

A recent study reported that the neoplasm or malignancy rate was not different among these architectural patterns of follicular lesions. ${ }^{15} \mathrm{~A}$ CNB cannot discriminate between a follicular carcinoma and a follicular adenoma because the diagnosis of these neoplasms requires examination of the entire thick fibrous capsule. However, in a subset of this category, focal nuclear atypia raises the possibility of a follicular variant of papillary carcinoma (Fig. 5). ${ }^{15,17,18} \mathrm{~A}$ follicular variant of papillary carcinoma is reportedly the most common malignancy found after surgery of thyroid nodules with a preoperative diagnosis of follicular neoplasm. $^{17,18}$

Histological findings of a follicular neoplasm on the CNB sample should be correlated with ultrasound imaging features. Typical ultrasound images of a follicular neoplasm show a wellcircumscribed, solid, ovoid or round mass with a surrounding halo or a hypoechoic rim around the thyroid nodule (Figs. 3,
5). ${ }^{19,20}$ The neoplasm can be hypoechoic, isoechoic, hyperechoic, or mixed. ${ }^{20}$ The sonographic halo corresponds with the fibrous capsule surrounding the follicular lesion (Figs. 3, 5). ${ }^{19,20}$ Focal cystic change can be present, but calcification is rare. ${ }^{20}$

\section{Suspicious for malignancy}

The "suspicious for malignancy" diagnosis is given when histologic features are strongly suspicious for malignancy, but they are insufficient for a definitive diagnosis of malignancy. In this category, a lesion may be suspicious for papillary thyroid carcinoma, suspicious for medullary thyroid carcinoma, suspicious for poorly differentiated thyroid carcinoma, suspicious for metastatic carcinoma, suspicious for lymphoma, or show other suspicious findings.

Ancillary immunohistochemical or molecular studies can be helpful for the diagnosis of CNB specimens with findings that are suspicious for malignancy. An immunostaining panel consisting of galectin-3, HBME1, cytokeratin 19, or CD56 is helpful in the diagnosis of lesions suspicious for papillary thyroid carcinoma (Fig. 6). ${ }^{21-23} \mathrm{~A}$ combination of at least two immunostaining markers is recommended to improve the diagnostic ac-

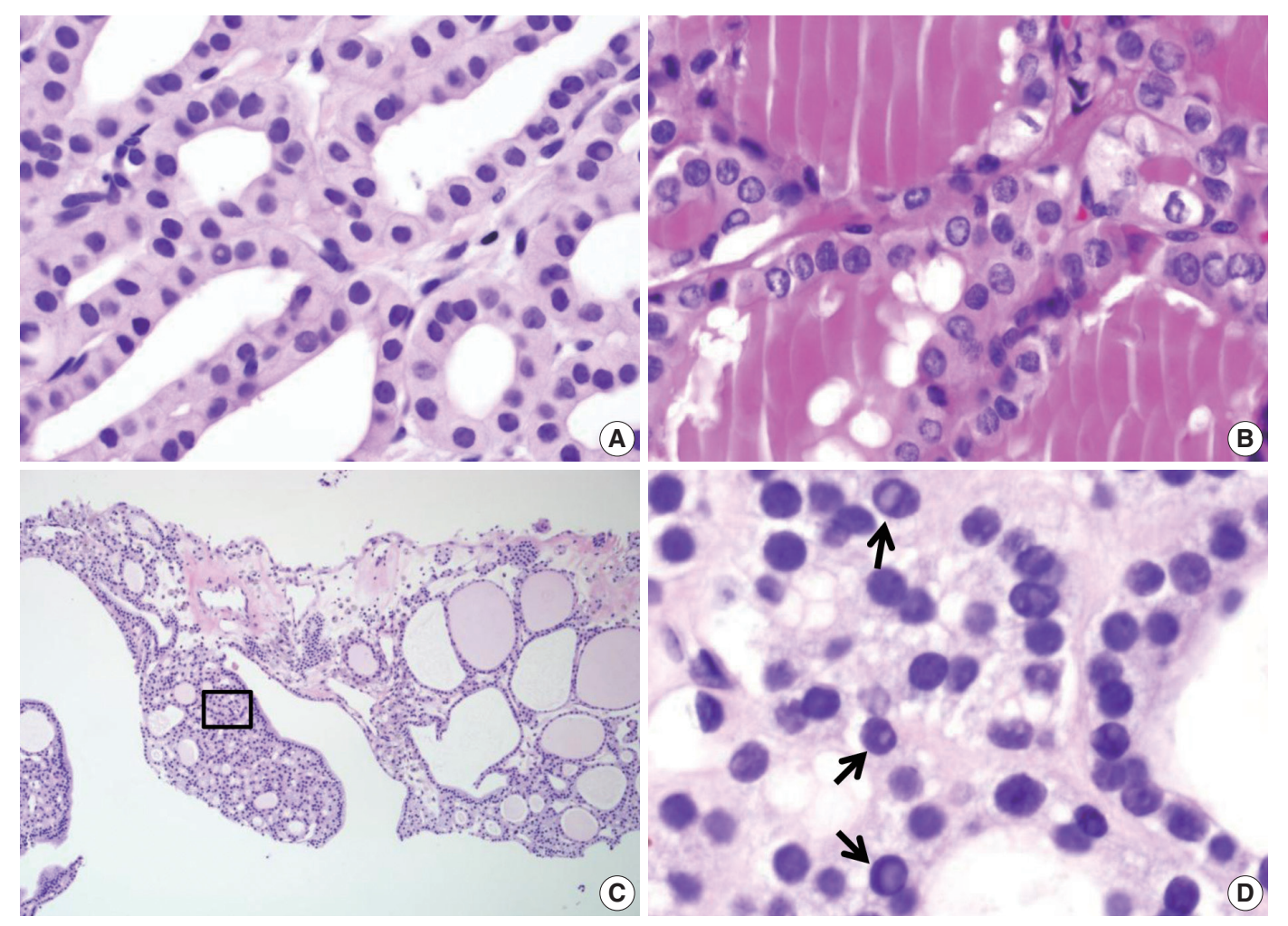

Fig. 8. Diagnostic pitfalls in thyroid core needle biopsy. Follicular cells are smaller and darker in core needle biopsies in comparison (A) to resected specimens (B). These images have been obtained from the same patient as those pictured in Fig. 6. (C) The core needle biopsy shows the histologic features of a benign follicular nodule. (D) The high-power view of the boxed area in Fig. $8 \mathrm{C}$ shows nuclear vacuoles that mimic intranuclear cytoplasmic pseudoinclusions in papillary carcinoma (arrows). 
curacy. $^{22}$ Presence of the BRAF V600E mutation, detected by molecular test or immunostaining, strongly suggests a diagnosis of the papillary carcinoma. ${ }^{21}$

A diagnosis of medullary thyroid carcinoma can be confirmed with positive immunostaining for calcitonin on a CNB specimen. A diagnosis of lymphoma can be rendered using immunophenotyping studies on a $\mathrm{CNB}$ specimen that is suspicious for lymphoma.

\section{Malignant}

Most thyroid malignancies, except for follicular carcinoma, have typical histologic features and are easily diagnosed as a malignancy on a $\mathrm{CNB}$ specimen. The following diagnoses are included in this category: papillary thyroid carcinoma (Fig. 7A, B), poorly differentiated carcinoma (Fig. 7C, D), undifferentiated (anaplastic) carcinoma, medullary thyroid carcinoma (Fig. 7E, F), lymphoma, and metastatic carcinoma.

\section{COMMON PITFALLS OF A DIAGNOSIS OF CORE NEEDLE BIOPSY}

The follicular cells in a CNB specimen appear smaller and show darker chromatin than in typical surgical specimens (Fig. 8A, B). Nuclear artifacts that mimic intranuclear cytoplasmic pseudoinclusions in papillary carcinoma may also be present in benign follicular cells (Fig. 8C, D). The artifactual vacuoles are irregularly shaped and appear pale on staining, whereas pseudoinclusions in papillary carcinoma are round and sharply delineated by the rim of the nuclear membrane.

\section{Conflicts of Interest}

No potential conflict of interest relevant to this article was reported.

\section{Acknowledgments}

The authors would like to thank the members of the Endocrine Pathology Study Group of the Korean Society of Pathologists for their valuable expertise and suggestions throughout this study. This research was supported by the Korean Society of Pathologists Grant 2013 and Basic Science Research Program through the National Research Foundation of Korea (NRF) funded by the Ministry of Science, ICT and future planning (2013R1A2A2A01068570).

\section{REFERENCES}

1. American Thyroid Association (ATA) Guidelines Taskforce on Thyroid Nodules and Differentiated Thyroid Cancer, Cooper DS, Doherty GM, et al. Revised American Thyroid Association management guidelines for patients with thyroid nodules and differentiated thyroid cancer. Thyroid 2009; 19: 1167-214.

2. Ali SZ. Thyroid cytopathology: Bethesda and beyond. Acta Cytol 2011; 55: 4-12.

3. Cibas ES, Ali SZ; NCI Thyroid FNA State of the Science Conference. The Bethesda System for Reporting Thyroid Cytopathology. Am J Clin Pathol 2009; 132: 658-65.

4. Lee SH, Kim MH, Bae JS, Lim DJ, Jung SL, Jung CK. Clinical outcomes in patients with non-diagnostic thyroid fine needle aspiration cytology: usefulness of the thyroid core needle biopsy. Ann Surg Oncol 2014; 21: 1870-7.

5. Hahn SY, Shin JH, Han BK, Ko EY, Ko ES. Ultrasonography-guided core needle biopsy for the thyroid nodule: does the procedure hold any benefit for the diagnosis when fine-needle aspiration cytology analysis shows inconclusive results? Br J Radiol 2013; 86: 20130007.

6. Trimboli P, Crescenzi A. Thyroid core needle biopsy: taking stock of the situation. Endocrine 2015; 48: 779-85.

7. Sung JY, Na DG, Kim KS, et al. Diagnostic accuracy of fine-needle aspiration versus core-needle biopsy for the diagnosis of thyroid malignancy in a clinical cohort. Eur Radiol 2012; 22: 1564-72.

8. Na DG, Kim JH, Sung JY, et al. Core-needle biopsy is more useful than repeat fine-needle aspiration in thyroid nodules read as nondiagnostic or atypia of undetermined significance by the Bethesda system for reporting thyroid cytopathology. Thyroid 2012; 22: 468-75.

9. Baek JH, Na DG, Lee JH, et al. Core needle biopsy of thyroid nodules: consensus statement and recommendations. J Korean Soc Ultrasound Med 2013; 32: 95-102.

10. Ha EJ, Baek JH, Lee JH, et al. Core needle biopsy can minimise the non-diagnostic results and need for diagnostic surgery in patients with calcified thyroid nodules. Eur Radiol 2014; 24: 1403-9.

11. Yeon JS, Baek JH, Lim HK, et al. Thyroid nodules with initially nondiagnostic cytologic results: the role of core-needle biopsy. Radiology 2013; 268: 274-80.

12. Trimboli P, Nasrollah N, Guidobaldi L, et al. The use of core needle biopsy as first-line in diagnosis of thyroid nodules reduces false negative and inconclusive data reported by fine-needle aspiration. World J Surg Oncol 2014; 12: 61.

13. Nasrollah N, Trimboli P, Rossi F, et al. Patient's comfort with and tolerability of thyroid core needle biopsy. Endocrine 2014; 45: 79-83.

14. Baloch ZW, LiVolsi VA. Our approach to follicular-patterned lesions of the thyroid. J Clin Pathol 2007; 60: 244-50. 
15. Min HS, Kim JH, Ryoo I, Jung SL, Jung CK. The role of core needle biopsy in the preoperative diagnosis of follicular neoplasm of the thyroid. APMIS 2014; 122: 993-1000.

16. Yassa L, Cibas ES, Benson CB, et al. Long-term assessment of a multidisciplinary approach to thyroid nodule diagnostic evaluation. Cancer 2007; 111: 508-16.

17. Bae JS, Choi SK, Jeon S, et al. Impact of NRAS mutations on the diagnosis of follicular neoplasm of the thyroid. Int J Endocrinol 2014; 2014: 289834.

18. Ustun B, Chhieng D, Van Dyke A, et al. Risk stratification in follicular neoplasm: a cytological assessment using the modified Bethesda classification. Cancer Cytopathol 2014; 122: 536-45.

19. Sillery JC, Reading CC, Charboneau JW, Henrichsen TL, Hay ID, Mandrekar JN. Thyroid follicular carcinoma: sonographic features of 50 cases. AJR Am J Roentgenol 2010; 194: 44-54.

20. Reading CC, Charboneau JW, Hay ID, Sebo TJ. Sonography of thyroid nodules: a "classic pattern" diagnostic approach. Ultrasound Q 2005; 21: 157-65.

21. Crescenzi A, Guidobaldi L, Nasrollah N, et al. Immunohistochemistry for $B R A F(\mathrm{~V} 600 \mathrm{E})$ antibody VE1 performed in core needle biopsy samples identifies mutated papillary thyroid cancers. Horm Metab Res 2014; 46: 370-4.

22. Alshenawy HA. Utility of immunohistochemical markers in diagnosis of follicular cell derived thyroid lesions. Pathol Oncol Res 2014; 20: 819-28.

23. El Demellawy D, Nasr AL, Babay S, Alowami S. Diagnostic utility of CD56 immunohistochemistry in papillary carcinoma of the thyroid. Pathol Res Pract 2009; 205: 303-9. 